\title{
Febrile respiratory illnesses in infancy and atopy are risk factors for persistent asthma and wheeze
}

\author{
M.M.H. Kusel*, T. Kebadze ${ }^{\#}$, S.L. Johnston ${ }^{\#}$, P.G. Holt* and P.D. Sly*,
}

ABSTRACT: Severe viral respiratory illnesses and atopy are risk factors for childhood wheezing and asthma. The aim of this study was to explore associations between severe respiratory infections and atopy in early childhood with wheeze and asthma persisting into later childhood.

147 children at high atopic risk were followed from birth to age 10 yrs. Data on all respiratory infections occurring in infancy were collected prospectively and viral aetiology ascertained. Atopy was measured by skin prick tests at 6 months, and 2 and 5 yrs. History of wheeze and doctordiagnosed eczema and asthma was collected regularly until 10 yrs of age.

At $10 \mathrm{yrs}, 60 \%$ of the cohort was atopic, $25.9 \%$ had current eczema, $18.4 \%$ current asthma and 20.4\% persistent wheeze. $35.8 \%$ experienced at least one lower respiratory infection (LRI) associated with fever and/or wheeze in first year of life. Children who had wheezy or, in particular, febrile LRI in infancy and were atopic by 2 yrs, were significantly more likely to have persistent wheeze (RR 3.51, 95\% Cl 1.83-6.70; $\mathbf{p}<0.001$ ) and current asthma (RR 4.92, 95\% $\mathrm{Cl} 2.59-9.36 ; p<0.001)$ at 10 yrs.

Severe viral respiratory infections in infancy and early atopy are risk factors for persistent wheeze and asthma. The strongest marker of the asthmatogenic potential of early life infections was concurrent fever. The occurrence of fever during respiratory illnesses is an important marker of risk for wheeze and asthma later in childhood, suggesting it should be measured in prospective studies of asthma aetiology.

KEYWORDS: Asthma, atopy, febrile infections, persistent wheeze, severe respiratory infections

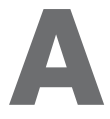
sthma in children remains the most common cause of emergency room visits and hospitalisation [1]. Despite intense research, asthma prevention strategies remain elusive, in part, because of its genetically complex and heterogeneous nature, which has several clinical phenotypes [2,3]. Although all phenotypes of childhood wheezing are of concern, persistent asthma and wheeze carry the heaviest toll on both the individual and healthcare resources. Thus, the early and precise identification of children at risk of persistent asthma and wheeze is necessary if early intervention strategies are to be effective.

Multiple risk factors for persistent wheeze and asthma have been identified, including allergic rhinitis, eczema and atopy, particularly when sensitisation occurs early in childhood [4]. One of the strongest risk factors appears to be viral respiratory infections, which are common in

early childhood and frequently associated with wheezing symptoms and subsequent asthma diagnosis [5]. Respiratory syncytial virus (RSV)associated lower respiratory illnesses (LRIs) have been reported to be risk factors for frequent wheeze in early childhood, especially in those requiring hospitalisation, but this risk diminished with increasing age [6].

Previously, RSV and human metapneumovirus (HMPV) have been implicated as major aetiological agents for LRI [7, 8], but human rhinoviruses (HRVs) are gaining recognition as significant LRI pathogens [9-13]. Infants hospitalised for HRV-induced wheezing in infancy were found to have increased risk of asthma at 6 yrs in one of the first studies to report HRVs as inducers of early childhood wheezing [9]. A cohort of 118 infants hospitalised for wheezy illnesses found HRV-associated wheezing was a significant risk

This article has supplementary material available from www.erj.ersjournals.com Earn CME accreditation by answering questions about this article. You will find these at the back of the printed copy of this issue or online at www.erj.ersjournals.com/misc/cmeinfo.xhtml

\section{AFFILIATIONS}

*Telethon Institute for Child Health Research, Perth, and

'Queensland Children's Medical Research Institute, University of Queensland, Queensland, Australia. \# National Heart and Lung Institute, Imperial College, London, UK

CORRESPONDENCE

M.M.H. Kusel

Division of Clinical Sciences

Telethon Institute for Child

Health Research

P0 Box 855

Perth 6872

Australia

E-mail: mercik@ichr.uwa.edu.au

Received:

Dec 142010

Accepted after revision:

Aug 052011

First published online:

Sept 152011 
factor for recurrent wheezing [10]. The oldest child at outcome was 35 months and further follow-up will determine whether the wheezing persisted. LEMANSKE et al. [11] investigated a high-risk cohort and found HRV-wheezing illnesses in infancy to be associated with pre-school wheezing. Similarly, HRV- or RSVassociated wheeze in the first 3 yrs of life was associated with asthma at 6 yrs [12]. Patterns of wheezy illnesses appear to be set by 6 yrs and do not appear to change at age 16 yrs in children presenting with asthma-like symptoms early in life [14].

Evidence is accumulating that these risk factors do not act alone, with the highest risk for development of persistent asthma observed in children who experience both early atopic sensitisation and severe LRIs during infancy [5]. In this context, we have previously demonstrated that children who experienced HRV- or RSV-associated LRI in their first year of life were more likely to report current wheeze and asthma at 5 yrs, and this effect was confined to children who were sensitised by 2 yrs [15]. This high-risk cohort has recently completed their 10-yr follow-up, and we report the effects of respiratory illnesses in the first year and early atopic sensitisation on the persistence of wheeze and asthma to 10 yrs of age.

\section{METHODS}

The study subjects were the 147 (74.2\%) children who completed the 10-yr assessment from the 198 infants seen at 5 yrs of age in a previously described prospective birth cohort [15]. The children who were at high risk of atopy (at least one parent with doctor-diagnosis of eczema, hayfever or asthma), were recruited and followed from birth until 10 yrs of age. Close follow-up of all respiratory illnesses was performed through the use of daily diaries to record symptoms of acute respiratory illnesses (ARIs) and fortnightly follow-up telephone calls until resolution of symptoms. The diary recorded symptom duration, including runny/blocked nose, fever (temperature $>38^{\circ} \mathrm{C}$ taken with digital thermometers), cough and wheeze. These data were confirmed during follow-up calls and information about any physician visit and doctor diagnoses. Home visits to collect nasopharyngeal aspirates (NPAs) were performed within $48 \mathrm{~h}$ of illness notification. NPAs, including two control samples (one taken in winter and another in summer when the child was well), were frozen at $-80^{\circ} \mathrm{C}$ and subsequently analysed by RT-PCR for HRVs, other picornaviruses (coxsackie, echo and enteroviruses), RSV, influenza A and B, coronaviruses 229E and OC43, parainfluenza viruses 1-3, adenoviruses, HMPV, Chlamydia pneumoniae and Mycoplasma pneumoniae, as previously described [16].

Atopy was determined by skin prick tests (SPT) at 6 months, and 2, 5 and 10 yrs to a panel of seven allergens (cow's milk, egg white, ryegrass, alternaria, aspergillus, house dust mite and cat dander). Histamine was the positive control and normal saline the negative control. Wheal size was read after $15 \mathrm{~min}$ and a wheal $\geqslant 2 \mathrm{~mm}$ (for tests at 6 months and $2 \mathrm{yrs}$ ) or $\geqslant 3 \mathrm{~mm}$ (at 5 and $10 \mathrm{yrs}$ ) greater than saline control was considered positive. Similar SPTs were performed on the parents and a wheal size $\geqslant 3 \mathrm{~mm}$ was considered positive.

\section{Classification of ARIs}

Episodes of ARI were classified as upper and lower respiratory illnesses (URIs and LRIs, respectively) based on information derived from diary cards and telephone contacts.

\section{Upper respiratory illnesses}

URI was defined as episodes of runny/blocked nose or cough in absence of other respiratory symptoms (no tachypnoea, difficulty breathing, wheeze or rattly chest).

\section{Lower respiratory illnesses}

LRI was defined as episodes associated with wheeze or rattly chest and/or evidence of respiratory distress. Rattle/rattly chest was defined as moist, wet noisy breath sounds from the child's chest and wheeze as audible, expiratory, high-pitched whistling sounds from parental or doctor's reports. Because wheeze is not easy to recognise, episodes were verified with the study doctor or family physician $(46 \%$ of ARIs were seen by family physicians). LRIs were classified as wheezy LRI and non-wheezy LRI based on the presence of wheeze.

\section{Febrile $A R I$}

Febrile infections was defined as ARI associated with fever (temperature $>38^{\circ} \mathrm{C}$ on two occasions $>1 \mathrm{~h}$ apart in the previous $48 \mathrm{~h}$ ).

\section{Febrile LRI}

Febrile LRI was defined as episodes of LRI with fever.

\section{Definitions}

Persistent wheeze was defined: as wheeze occurring between 0 and 3 yrs and continuing in the 12 months preceding the 10yr visit; asthma as doctor diagnosis of asthma ever; current asthma as asthma and persistent wheeze or asthma medication in the preceding 12 months; eczema as doctor diagnosis of eczema ever; and current eczema as eczema in the preceding 12 months.

\section{Statistical analysis}

Log-binomial regression models were used to estimate risk ratios for persistent wheeze and current asthma at $10 \mathrm{yrs}$. Multivariate models were adjusted for sex, breastfeeding, pet ownership, older siblings, second-hand tobacco smoke exposure and daycare attendance. All analyses were performed using STATA version 11.0 (Stata Corporation, College Station, TX, USA). p-values $<0.05$ were considered statistically significant.

Current data from 147 children ( $\sim 20 \%$ with current asthma and persistent wheeze at $10 \mathrm{yrs}$ ) provided an $80 \%$ power to detect a relative risk of $\geqslant 2.1$ at $p<0.05$ level of significance for association with current asthma (or persistent wheeze) for a risk factor with $>20 \%$ prevalence.

The ethics committee of King Edward Memorial and Princess Margaret Hospitals in Western Australia gave approval of the study, and fully informed parental consent was obtained for all subjects.

\section{RESULTS}

\section{Cohort characteristics}

$147(74.78 \%)$ of the 198 children who were seen at 5 yrs of age completed this $10 \mathrm{yr}$ follow-up. No significant differences were found in prevalence of atopy or wheeze/asthma at 5 yrs in the children who were seen at the 10-yr follow-up compared with those who were not involved in this visit. Information on 
cohort size and follow-up numbers is given in the online supplementary material.

The majority of the cohort was male (57.8\%). 38.8\% attended daycare in their first year of life. Only five (3.4\%) were exposed to maternal smoking in utero and $12.9 \%$ to second-hand smoke in the first 10 yrs. Prevalence of parental atopic disease was high, with hayfever and asthma the more common reported conditions. $83.0 \%$ of fathers and $82.3 \%$ of mothers were atopic, and $68.7 \%$ of the cohort had biparental atopy. $69.4 \%$ of the children were breastfed for $>26$ weeks.

\section{Prevalence of atopic conditions}

$49.0 \%$ reported doctor diagnosis of allergic rhinoconjunctivitis (ARC) (table 1). 77 (52.4\%) had eczema ever and 25.9\% current eczema (table 1). 59.9\% were atopic (food and aeroallergen sensitisation reported in online supplementary material). $36.1 \%$ had been diagnosed with asthma (table 1). Of these, $27(50.9 \%)$ had current asthma at 10 yrs and 30 (56.6\%) out of 53 had persistent wheeze. $66.7 \%$ of children with both current asthma (18 (66.7\%) out of 27$)$ and persistent wheeze (20 (66.7\%) out of 30) were found to be atopic by the age of 2 yrs.

\section{Frequency of episodes of respiratory illnesses}

In the first year of life, $73.5 \%$ had $\geqslant 3$ episodes of ARI, with the majority of these being URI (table 2 ). $52.4 \%$ of children experienced $\geqslant 3$ episodes of URI, $59.2 \% \geqslant 1$ episode of febrile ARI and $66 \% \geqslant 1$ episode of LRI (table 2). $26.5 \%$ had at least one episode of wheezy LRI and $26.5 \%$ had at least one episode of febrile LRI.

\section{Predictors for current asthma and persistent wheeze at 10 yrs}

Presence of eczema at 5 yrs and 10 yrs and ARC by 5 yrs were significantly associated with current asthma at 10 yrs (table 3). These characteristics and biparental atopy were also found to be significant for persistent wheeze at $10 \mathrm{yrs}$ (table 3).

Atopy at 2 yrs, 5 yrs and those with persistent atopy were found to have $>2.5$ times the risk of current asthma at $10 \mathrm{yrs}$ (table 3). This risk for current asthma increased substantially for children who had positive SPT at 10 yrs (risk ratio (RR) 17.14; 95\%CI 2.39-122.83; $\mathrm{p}=0.005$ ) (table 3). Similar significant associations were also found for persistent wheeze at $10 \mathrm{yrs}$, in particular if they were atopic at 10 yrs (RR 3.30, 95\% CI 1.348.11; $\mathrm{p}=0.009$ ) (table 3).

Children who wheezed in the first 3 yrs of life (RR 3.79, 95\% CI $1.52-9.46 ; \mathrm{p}=0.004)$, and those who wheezed throughout the first 5 yrs (RR 7.48, 95\% CI 3.73-15.00; $p<0.001$ ) were significantly more likely to have current asthma as well as persistent wheeze (table 3). No significant associations were found between URI and wheeze or asthma at $10 \mathrm{yrs}$.

In contrast, children who had experienced HRV-associated wheezy LRI in their first year of life were more likely to have persistent wheeze at 10 yrs (RR 1.99, 95\%CI 1.04-3.84; $\mathrm{p}=0.04$ ) (table 3). There was a similar trend for current asthma, but this association failed to reach statistical significance. Stronger associations were seen with early febrile LRI. Children who experienced at least one episode of febrile LRI in their first year of life had more than twice the risk of current asthma (RR 2.57, 95\% CI 1.33-4.98; $\mathrm{p}=0.005)$ and persistent wheeze (RR 2.12,

\section{TABLE 1 Characteristics of the cohort}

\section{Male}

Number of older siblings

0

1

$\geqslant 2$

\section{Pet ownership}

Childcare attendance in first year of life

None

First 6 months of age

7-12 months of age

Antenatal smoking

Environmental tobacco smoke exposure

Maternal history

Asthma

Hayfever

Eczema

Atopy

Paternal history

Asthma

Hayfever

Eczema

Atopy

Biparental atopy

Duration of breastfeeding

0-6 weeks

$7-12$ weeks

13-20 weeks

21-26 weeks

$>26$ weeks

Atopic conditions at $10 \mathrm{yrs}$

Allergic rhinoconjunctivitis

Eczema ever

Current eczema

Atopy

Doctor diagnosis of asthma (ever)

Current asthma

Persistent wheeze

$85(57.8)$

$76(51.7)$

$41(27.9)$

$30(20.4)$

108 (73.5)

90 (61.2)

20 (13.6)

$37(25.2)$

5 (3.4)

$19(12.9)$

$97(66.0)$

$132(89.8)$

$61(41.5)$

$121(82.3)$

62 (42.2)

$93(63.3)$

22 (15.0)

$122(83.0)$

$101(68.7)$

$11(7.5)$

$16(10.9)$

$11(7.5)$

$7(4.8)$

$102(69.4)$

$72(49.0)$

77 (52.4)

$38(25.9)$

$88(59.9)$

$53(36.1)$

27 (18.4)

$30(20.4)$

Data are presented as $\mathrm{n}(\%)$

$95 \%$ CI 1.14-3.95; $\mathrm{p}=0.018$ ) at $10 \mathrm{yrs}$. This relationship between febrile LRI and current asthma and persistent wheeze appeared strongest if they were associated with RSV (table 3).

\section{Predictors for persistent wheeze and current asthma in relation to time of atopic sensitisation}

Children who experienced any febrile respiratory infection had increased likelihood of current asthma (table 4). This was, however, only seen in the group of children who were found to be sensitised by 2 yrs of age. This association increased more than two-fold for current asthma (RR 4.92, 95\%CI 2.59-9.36; $\mathrm{p}<0.001$ ) (table 4) if the febrile infection involved the lower respiratory tract. There was also an increased association for febrile infections and persistent wheeze (RR 2.29, 95\% CI 1.20$4.40 ; \mathrm{p}<0.0012)$ and, similarly, this risk increased for febrile LRI (RR 3.51, 95\% CI 1.83-6.70; p<0.001) (table 5). In contrast, wheezy LRIs in the first year of life were not significantly associated with current asthma or persistent wheeze at age 
TABLE 2 Frequency of different types of acute respiratory illnesses (ARIs) in the first year of life

\begin{tabular}{lcccccc} 
Number of episodes & ARI & URI & Febrile ARI & LRI & Wheezy LRI & Febrile LRI \\
\hline $\mathbf{0}$ & $5(3.4)$ & $17(11.6)$ & $60(40.8)$ & $50(34.0)$ & $108(73.5)$ & $108(73.5)$ \\
$\mathbf{1}$ & $14(9.5)$ & $24(16.3)$ & $48(32.7)$ & $45(30.6)$ & $25(17.0)$ & $39(26.5)$ \\
$\mathbf{2}$ & $20(13.6)$ & $29(19.7)$ & $29(19.7)$ & $26(17.7)$ & $13(8.8)$ & 0 \\
$\mathbf{3}$ & $128(73.5)$ & $67(52.4)$ & $10(6.8)$ & $26(17.7)$ & $1(0.7)$ & 0 \\
\hline
\end{tabular}

Data are presented as n (\%). URI: upper respiratory illness; LRI: lower respiratory illness.

10 yrs (table 3). HRV-associated wheezy LRI were significantly associated with current asthma (table 4) and persistent wheeze (table 5) in those atopic after 2 yrs of age.

\section{DISCUSSION}

In an earlier publication on the 5-yr findings from this birth cohort of high risk children, we reported that current wheeze

TABLE 3 Adjusted risk ratios ${ }^{\#}$ for various early predictors for current asthma and persistent wheeze at 10 yrs

\begin{tabular}{|c|c|c|c|c|c|}
\hline Characteristic & Subjects $\mathbf{n}$ & Current asthma at $10 \mathrm{yrs}$ & p-value & Persistent wheeze at 10 yrs & p-value \\
\hline Total subjects $n$ & & 27 & & 30 & \\
\hline Male sex & 85 & $1.46(0.70-3.03)$ & 0.311 & $1.09(0.57-2.10)$ & 0.787 \\
\hline Pet ownership (first 5 yrs) & 80 & $0.78(0.39-1.54)$ & 0.470 & $1.10(0.57-2.09)$ & 0.782 \\
\hline Antenatal smoking & 5 & 0 & & $0.98(0.16-5.82)$ & 0.982 \\
\hline Day care in first year & 42 & $0.71(0.31-1.64)$ & 0.429 & $1.25(0.64-2.44)$ & 0.514 \\
\hline Paternal asthma & 41 & $1.29(0.63-2.64)$ & 0.481 & $1.11(0.55-2.22)$ & 0.772 \\
\hline Paternal atopy & 122 & $2.56(0.65-10.13)$ & 0.180 & $2.87(0.73-11.27)$ & 0.131 \\
\hline Maternal asthma & 74 & $1.43(0.72-2.88)$ & 0.309 & $1.48(0.77-2.85)$ & 0.241 \\
\hline Maternal atopy & 121 & $1.72(0.56-5.28)$ & 0.344 & $1.93(0.63-5.90)$ & 0.246 \\
\hline Biparental atopy & 101 & $2.62(0.96-7.14)$ & 0.060 & $2.96(1.10-7.99)$ & 0.032 \\
\hline Eczema at 5 yrs & 49 & $2.15(1.10-4.22)$ & 0.025 & $1.53(0.81-2.89)$ & 0.190 \\
\hline Eczema at 10 yrs & 38 & $3.59(1.85-6.96)$ & $<0.001$ & $2.87(1.55-5.30)$ & 0.001 \\
\hline Allergic rhinoconjunctivitis by 5 yrs & 59 & $3.54(1.66-7.55)$ & 0.001 & $2.24(1.17-4.29)$ & 0.015 \\
\hline \multicolumn{6}{|l|}{ Atopy } \\
\hline Atopic at 6 months & 31 & $1.58(0.76-3.25)$ & 0.219 & $1.60(0.82-3.14)$ & 0.169 \\
\hline Atopic at 2 yrs & 63 & $2.67(1.28-5.54)$ & 0.008 & $2.67(1.34-5.29)$ & 0.005 \\
\hline Atopic at $5 \mathrm{yrs}$ & 56 & $3.04(1.45-6.34)$ & 0.003 & $2.10(1.11-3.99)$ & 0.023 \\
\hline Early atopy (by 2 yrs, not at 5 yrs) & 34 & $0.95(0.42-2.16)$ & 0.902 & $1.21(0.59-2.46)$ & 0.602 \\
\hline Atopic only at 5 yrs & 22 & $1.62(0.74-3.56)$ & 0.227 & $1.14(0.49-2.65)$ & 0.767 \\
\hline Persistent atopy (atopic at 2 and 5 yrs) & 35 & $2.56(1.33-4.94)$ & 0.005 & $2.13(1.14-3.98)$ & 0.017 \\
\hline Atopic at $10 \mathrm{yrs}$ & 88 & $17.14(2.39-122.83)$ & 0.005 & $3.30(1.34-8.11)$ & 0.009 \\
\hline \multicolumn{6}{|l|}{ Wheeze phenotype } \\
\hline Wheeze ever $0-3$ yrs & 79 & $3.79(1.52-9.46)$ & 0.004 & $1.72(0.87-3.42)$ & 0.121 \\
\hline Wheeze $3-5$ yrs & 55 & $7.36(2.96-18.32)$ & $<0.001$ & $3.90(1.93-7.91)$ & $<0.001$ \\
\hline Persistent wheeze & 31 & $7.48(3.73-15.00)$ & $<0.001$ & $3.74(2.06-6.79)$ & $<0.001$ \\
\hline \multicolumn{6}{|l|}{ ARI in first year } \\
\hline Wheezy LRI at $1 \mathrm{yr}$ & 39 & $1.17(0.56-2.44)$ & 0.684 & $1.19(0.60-2.37)$ & 0.626 \\
\hline Wheezy LRI + RSV & 10 & $1.10(0.30-3.98)$ & 0.889 & $1.52(0.56-4.16)$ & 0.413 \\
\hline Wheezy LRI + HRV & 26 & $1.63(0.77-3.45)$ & 0.202 & $1.99(1.04-3.84)$ & 0.039 \\
\hline Wheezy LRI with HRV or RSV & 30 & $1.37(0.64-2.92)$ & 0.423 & $1.67(0.86-3.27)$ & 0.133 \\
\hline Febrile LRI at $1 \mathrm{yr}$ & 39 & $2.57(1.33-4.98)$ & 0.005 & $2.12(1.14-3.95)$ & 0.018 \\
\hline Febrile LRI + RSV & 10 & $3.11(1.50-6.45)$ & 0.002 & $2.74(1.34-5.60)$ & 0.006 \\
\hline Febrile LRI + HRV & 13 & $1.79(0.73-4.39)$ & 0.202 & $2.06(0.95-4.47)$ & 0.067 \\
\hline Febrile LRI + HRV or RSV & 20 & $2.67(1.36-5.27)$ & 0.004 & $2.31(1.20-4.46)$ & 0.013 \\
\hline Any wheezy or febrile LRI at $1 \mathrm{yr}$ & 53 & $1.42(0.72-2.80)$ & 0.314 & $1.55(0.82-2.92)$ & 0.174 \\
\hline
\end{tabular}

Data are presented as risk ratio $(95 \% \mathrm{Cl})$, unless otherwise stated. ARI: acute respiratory illness; LRI: lower respiratory illness; HRV: human rhinovirus; RSV: respiratory syncytial virus. \#: adjusted for sex, breastfeeding, pet ownership, older siblings, second-hand tobacco smoke exposure and daycare attendance. Bold indicates statistical significance. 
TABLE 4 Adjusted risk ratios ${ }^{\#}$ for infections and current asthma $(n=27)$ at 10 yrs in relation to age of sensitisation

\begin{tabular}{|c|c|c|c|c|}
\hline Subjects $n$ & 69 & & 22 & \\
\hline \multicolumn{5}{|l|}{ Infections in first year } \\
\hline Any febrile infections & $2.28(1.16-4.46)$ & 0.017 & $1.91(0.75-4.85)$ & 0.176 \\
\hline Any febrile URI & $1.22(0.54-2.72)$ & 0.633 & $2.01(0.82-4.92)$ & 0.126 \\
\hline Any wheezy or febrile LRI & $2.92(1.45-5.88)$ & 0.003 & $0.61(0.09-3.97)$ & 0.601 \\
\hline Any wheezy LRI & $1.41(0.60-3.33)$ & 0.429 & $1.50(0.42-5.30)$ & 0.530 \\
\hline Any wheezy LRI with fever & $2.30(0.98-5.43)$ & 0.057 & $2.33(0.41-13.07)$ & 0.338 \\
\hline Any wheezy LRI without fever & $1.61(0.70-3.71)$ & 0.266 & $1.12(0.19-6.55)$ & 0.903 \\
\hline Any wheezy LRI associated with HRV or RSV & $1.36(0.54-3.43)$ & 0.519 & $2.24(0.75-6.64)$ & 0.146 \\
\hline
\end{tabular}

Data are presented as risk ratio $(95 \% \mathrm{Cl})$, unless otherwise stated. ARI: acute respiratory illnesses; URI: upper respiratory illness; LRI: lower respiratory illness; HRV: human rhinoviruses; RSV: respiratory syncytial virus. ${ }^{*}$ : adjusted for sex, breastfeeding, pet ownership, older siblings, second-hand tobacco smoke exposure and daycare attendance. Bold indicates statistical significance.

and/or asthma at outcome age was significantly associated with either wheezy or febrile LRI during the first year of life [15]. Moreover, these associations were observed in relation to infections associated with both RSV and HRV, although the effects were strongest for HRV, consistent with what has been reported for the Childhood Origins of Asthma Study (COAST) cohort [11]. We additionally found the associations of these infections with asthma were strongest in the subset of children who developed atopic sensitisation before the age of 2 yrs [15]. This suggested that interactions may occur between inflammatory pathways related to anti-viral and allergen-specific immunity, which contribute towards the subsequent development of the asthma phenotype [5]. Children who experienced more than one episode of wheeze associated with LRI during their first year had the highest prevalence of wheeze-related outcomes at 5 yrs. The findings reported here for the same cohort at $10 \mathrm{yrs}$ contain many similarities but also some significant differences.

The waning of the association between wheezing LRI during the first year of life and subsequent asthma-related phenotypes ascertained at $10 \mathrm{yrs}$ versus $5 \mathrm{yrs}$ is of particular interest. As noted above, early wheeze is generally invoked as the "gold standard" in relation to early identification of children at high risk of subsequent persistent asthma; depending on the

TABLE 5 Adjusted risk ratios ${ }^{\#}$ for infections and persistent wheeze $(n=30)$ at 10 yrs in relation to age of sensitisation

\begin{tabular}{|c|c|c|c|c|}
\hline Characteristic & Atopic by 2 yrs & p-value & Atopic after 2 yrs & p-value \\
\hline Subjects $\mathbf{n}$ & 69 & & 22 & \\
\hline Whole population regardless of ARI history & $2.35(1.13-4.90)$ & 0.022 & $1.22(0.52-2.86)$ & 0.652 \\
\hline \multicolumn{5}{|l|}{ Infections in first year } \\
\hline Any febrile infections & $2.29(1.20-4.40)$ & 0.012 & $2.12(0.83-5.38)$ & 0.115 \\
\hline Any febrile URI & $1.11(0.50-2.49)$ & 0.795 & $2.58(1.04-6.43)$ & 0.041 \\
\hline Any LRI with fever & $3.51(1.83-6.70)$ & $<0.001$ & $1.13(0.17-7.63)$ & 0.903 \\
\hline Any LRI without fever & $2.11(1.11-3.99)$ & 0.022 & $1.44(0.52-3.99)$ & 0.481 \\
\hline Any wheezy or febrile LRI & $2.64(1.39-5.02)$ & 0.003 & $0.67(0.10-4.54)$ & 0.685 \\
\hline Any wheezy LRI & $1.60(0.73-3.47)$ & 0.238 & $1.74(0.46-6.58)$ & 0.412 \\
\hline Any wheezy LRI with fever & $2.05(0.88-4.79)$ & 0.096 & $2.31(0.32-16.73)$ & 0.406 \\
\hline Any wheezy LRI without fever & $1.81(0.84-3.86)$ & 0.128 & $1.43(0.24-8.51)$ & 0.696 \\
\hline Any wheezy LRI associated with HRV or RSV & $2.03(0.97-4.27)$ & 0.061 & $2.38(0.70-8.11)$ & 0.166 \\
\hline Any wheezy LRI associated with HRV & $2.03(0.97-4.27)$ & 0.061 & $3.46(1.01-11.82)$ & 0.048 \\
\hline Any wheezy LRI associated with RSV & $2.80(0.93-8.46)$ & 0.068 & 0 & \\
\hline
\end{tabular}

Data are presented as risk ratio $(95 \% \mathrm{Cl})$, unless otherwise stated. ARI: acute respiratory illnesses; URI: upper respiratory illness; LRI: lower respiratory illness; HRV: human rhinoviruses; RSV: respiratory syncytial virus. * : adjusted for sex, breastfeeding, pet ownership, older siblings, second-hand tobacco smoke exposure and daycare attendance. Bold indicates statistical significance. 
breadth and depth of the clinical and immunological assessments made within individual studies, this variable has been associated with very high prevalence of asthma in midchildhood [12]. Indeed our data in the present cohort show a similar picture (table 3), but only if wheeze is considered in isolation. When early atopic sensitisation is taken into account, as for the 5-yr outcomes [15], infection-associated risk at $10 \mathrm{yrs}$ is once again restricted to the early onset atopics. Moreover, the infections that are associated with maximum asthma risk in this target group are those associated with fever as opposed to wheeze (tables 3-5).

This finding echoes earlier reports from MARTINEZ et al. [2] from the Tucson cohort, which indicated that much of the wheeze encountered during infancy is transient ("transient early wheeze $\mathrm{e}^{\prime \prime}$ ) and may be a result of small airway size, which resolves over time with normal lung growth. Due to the small calibre of their airways at baseline, these subjects have a tendency to wheeze in response to even relatively mild stimuli that cause only small degrees of airway narrowing [2] and, in community studies, it is difficult to distinguish these subjects from those wheezing as a result of more intense airway inflammation. We have argued previously on the basis of findings from multiple studies that severe airways inflammation during the early postnatal phase of rapid lung growth has potential to disturb underlying tissue differentiation programmes responsible for establishing normal lung function, and, furthermore, that the sequelae of such disturbances can "track" over time and manifest in later life as abnormal respiratory functions associated with the asthma phenotype $[5,17]$.

Our current findings are consistent with the hypothesis that febrile responses at the time of infection spreading to the lower respiratory tract may constitute a more reliable marker of the type of LRI that is associated with asthma development than wheeze. The consistent findings in our 5- and 10-yr outcome data that such events are much more common in children with early atopic sensitisation may be explicable on the basis of our recent findings on viral-induced acute asthma exacerbations resulting in hospitalisation. As noted in multiple earlier studies [5], this high asthma susceptibility phenotype is restricted almost exclusively to children sensitised to perennial aeroallergens, and our recent studies [18] suggest the underlying mechanism is immunoglobulin (Ig) E-dependent and involves a T-helper cell type 2-associated inflammatory cascade in which FcER1 $\alpha+-$ activated myeloid cells, which are recruited into the virusinduced inflammatory response in the lower respiratory tract, play a central role. The prominence in these viral-induced responses of activated myeloid cells, which are a major source of interleukin (IL)-1 and IL-18 (note in this context the recent genome-wide association study identifying IL1RL1/IL18R1 variants in association with asthma risk [19]), provides a plausible explanation for the presence of fever symptoms.

Our present study is not the first to report an association between fever during respiratory infections in children and risk for subsequent asthma. A large random survey of 5-7- and 9-11-yrold German children participating in International Study of Asthma and Allergies in Childhood (ISAAC) Phase II study found retrospectively collected parental reports of repeated episodes of fever and antibiotic treatment in early life were strongly associated with increased asthma prevalence and current wheeze at school age [20]. Similar positive associations were found for hayfever and eczema. Because the information about the number of febrile episodes occurring in the first year of life and antibiotics taken in the first 3 yrs was collected retrospectively, it may be that parents of symptomatic children were more likely to recall more severe respiratory infections, that is, those associated with fever or those requiring antibiotics. In our prospective study, we found that antibiotics per se were not risk factors for asthma or atopy at 5 yrs [21]. In another study, 2-16-yrold children attending an asthma clinic who used acetaminophen for fever in the preceding 12 months had increased risk of severe asthma [22]. No information was available on the causes of fever. Additionally, ISAAC Phase III reported paracetamol use for fever in year1 was associated with asthma symptoms at 6-7 yrs [23]. Similar associations were reported for rhinoconjunctivitis and eczema. The authors concluded that paracetamol was a risk factor for development of these conditions. It is plausible that the occurrence of fever, for which paracetamol has been used, is the underlying risk factor and the observed associations may be a result of confounding by indication.

It is also pertinent to note the association between early severe LRI and asthma at age 5 yrs [15] and (as shown here) $10 \mathrm{yrs}$ is not restricted to HRV but also includes RSV, particularly febrile RSV (table 3). This is consistent with findings in 11-yrolds from the Tucson cohort [6], but is at variance with data from the COAST cohort [11]. However, a notable feature of the present study was physician assessment of study subjects and NPA collection at every reported incidence of respiratory infection regardless of assumed severity. This is in contrast to the COAST study where nasal lavage for viral identification was carried out only when the child had a moderate-to-severe respiratory illness, as classified by a symptom score card [12].

Although the authors acknowledge the small size of this study population is a limitation of the study, we have collected comprehensive information about the nature of each ARI, including viral aetiology on all respiratory illnesses experienced by the children in their first year of life.

Our findings suggest that our unbiased approach might uncover associations between host responses to infection exemplified by fever and subsequent asthma pathogenesis, and the approach merits further testing in other prospective cohort studies.

In summary, prospectively collected data from our birth cohort of children at high risk of developing atopy and asthma show severe LRI associated with fever $>38^{\circ} \mathrm{C}$ are major risk factors for current asthma at 10 yrs of age, particularly in children who develop allergic sensitisation to aeroallergens by 2 yrs of age. This indicates an underlying IgE-dependent process occurring within the lungs, which may be related to that recently demonstrated in viral-associated asthma exacerbations [18]. These data suggest that fever should be objectively measured in cohort studies of asthma aetiology.

The authors thank J. Park for statistical assistance.

\section{SUPPORT STATEMENT}

This project has been supported by a National Health and Medical Research, Council Grant (Australia) and by the British Lung Foundation/Severin Wunderman, Family Foundation Programme (grant $\mathrm{P} 00 / 2$ ). 


\section{STATEMENT OF INTEREST}

None declared.

\section{REFERENCES}

1 Poulos L, Toelle B, Marks G. The burden of asthma in children: an Australian perspective. Paediatr Respir Rev 2005; 6: 20-27.

2 Martinez FD, Wright AL, Taussig LM, et al. Asthma and wheezing in the first six years of life. N Engl J Med 1995; 332: 133-138.

3 Brand P, Baraldi E, Bisgaard H, et al. Definition, assessment and treatment of wheezing disorders in preschool children: an evidence-based approach. Eur Respir J 2008; 32: 1096-1110.

4 Illi S, von Mutius E, Lau S, et al. Perennial allergen sensitisation early in life and chronic asthma in children: a birth cohort study. Lancet 2006; 368: 763-770.

5 Sly P, Boner AL, Björksten B, et al. Early identification of atopy in the prediction of persistent asthma in children. Lancet 2008; 372: 1100-1106.

6 Stein R, Sherrill D, Morgan WJ, et al. Respiratory syncytial virus in early life and risk of wheeze and allergy by age 13 years. Lancet 1999; 354: 541-545.

7 Gern J. Viral and bacterial infections in the development and progression of asthma. J Allergy Clin Immunol 2000; 105: 497-502.

8 García-García M, Calvo C, Casas I, et al. Human metapneumovirus bronchiolitis in infancy is an important risk factor for asthma at age 5. Pediatr Pulmonol 2007; 42: 458-464.

9 Kotaniemi-Syrjänen A, Vainionpää R, Reijonen TM, et al. Rhinovirus-induced wheezing in infancy - the first sign of childhood asthma? J Allergy Clin Immunol 2003; 111: 66-71.

10 Lehtinen $\mathrm{P}$, Ruohola A, Vanto $\mathrm{T}$, et al. Prednisolone reduces recurrent wheezing after a first wheezing episode associated with rhinovirus infection or eczema. J Allegy Clin Immunol 2007; 119: 570-575.

11 Lemanske RF Jr, Jackson DJ, Gangnon RE, et al. Rhinovirus illnesses during infancy predict subsequent childhood wheezing. J Allergy Clin Immunol 2005; 116: 571-577.

12 Jackson D, Gangnon RE, Evans MD, et al. Wheezing rhinovirus illnesses in early life predict asthma development in high risk children. Am J Respir Crit Care Med 2008; 178: 667-672.
13 Kusel MMH, de Klerk NH, Holt PG, et al. Role of rhinoviruses in acute upper and lower respiratory tract illness in the first year of life: a birth cohort study. Pediatr Infect Dis J 2006; 25: 680-686.

14 Morgan W, Stern DA, Sherrill DL, et al. Outcome of asthma and wheezing in the first 6 years of life: follow-up through adolescence. Am J Respir Crit Care Med 2005; 172: 1253-1258.

15 Kusel M, de Klerk NH, Kebadze T, et al. Early life respiratory viral infections, atopic sensitisation and risk of subsequent development of persistent asthma. J Allergy Clin Immunol 2007; 119: 1105-1110.

16 Johnston NW, Johnston SL, Duncan JM, et al. The September epidemic of asthma exacerbations in children: a search for etiology. J Allergy Clin Immunol 2005; 115: 132-138.

17 Holt P, Upham J, Sly P. Contemporaneous maturation of immunological and respiratory functions during early childhood: implications of development of asthma prevention strategies. J Allegy Clin Immunol 2005; 116: 16-24.

18 Subrata L, Bizzintino J, Mamessier E, et al. Interactions between innate antiviral and atopic immunoinflammatory pathways precipitate and sustain asthma exacerbations in children. J Immunol 2009; 183: 2793-2800.

19 Moffatt M, Gut IG, Demenais F, et al. A large-scale, consortiumbased genomewide association study of asthma. N Engl J Med 2010; 363: 1211-1221.

20 von Mutius E, Illi S, Hirsch T, et al. Frequency of infections and risk of asthma, atopy and airway hyperresponsiveness in children. Eur Respir J 1999; 14: 4-11.

21 Kusel MMH, de Klerk N, Holt PG, et al. Antibiotic use in the first year of life and risk of atopic disease in early childhood. Clin Exp Allergy 2008; 38: 1921-1928.

22 Martinez C, Sossa M, Goss C. Factors associated with severe disease in a population of asthmatic children of Bogota, Colombia. J Asthma 2008; 45: 141-147.

23 Beasley R, Clayton T, Crane J, et al. Association between paracetamol use in infancy and childhood, and risk of asthma, rhinoconjunctivitis, and eczema in children aged 6-7 years: analysis from phase three of the ISAAC programme. Lancet 2008; 372: 1039-1048. 